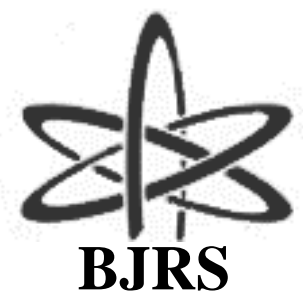

BRAZILIAN JOURNAL

$\mathrm{OF}$

RADIATION SCIENCES

03-01 (2015) 01-08

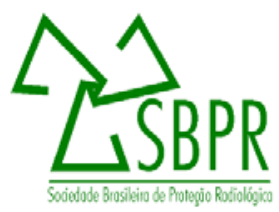

\title{
Amostragem de ar de alto fluxo para determinação de alfa emissores de meia vida longa: Monitoração de área em um depósito de material radioativo
}

\author{
W. S. Pereira ${ }^{1} \mathrm{e}^{2}$ e A. Kelecom ${ }^{3}$ \\ 1 - Laboratório de Monitoramento de Efluentes Líquidos - LAMEL, Curso de Pós-Graduação em Ciências do Meio \\ Ambiente, Universidade Veiga de Almeida (UVA), Rua Ibituruna, 108, Tijuca, Rio de Janeiro, Cep 20.271-020, Br. \\ 2 - Grupo Multidisciplinar de Radioproteção (GMR), Serviço de Radioproteção (SR), Fabrica do Combustível \\ Nuclear (FCN), Indústrias Nucleares do Brasil (INB), Caixa Postal: 83632, CEP: 25580-970 - Itatiaia - RJ \\ 3 - Laboratório de Radiobiologia e Radiometria Pedro Lopes dos Santos (LARARA-PLS), \\ Grupo de Estudos em Temas Ambientais (GETA), Universidade Federal Fluminense - UFF, C.P. 100436, \\ CEP 24.001-970, Niterói, RJ, Brazil.
}

\begin{abstract}
RESUMO
A Unidade de Tratamento de Minério (UTM) é uma mina e usina de urânio fechada, situada em Caldas, Minas Gerais, Brasil. Ela possui um depósito de material radioativo composto basicamente de torta II e mesotório. Esse material está estocado em seis galpões designados C-01, C-02, C-05, C-06, C-07 e C-09. O presente trabalho visa apresentar o programa de monitoração de área de alto fluxo e os resultados obtidos no ano de 2009. O limite derivado de concentração no ar foi de $0,25 \mathrm{~Bq} \cdot \mathrm{m}^{-3}$. As médias das concentrações de atividade no ano de 2009 foram: para C-01 $1,17 \mathrm{~Bq} \cdot \mathrm{m}^{-3}$; C-02 0,006 Bq $\cdot \mathrm{m}^{-3}$; C-05 1,98 Bq $\cdot \mathrm{m}^{-3}$; C-06 2,14 Bq $\cdot \mathrm{m}^{-3}$; C-07 0,34 Bq $\cdot \mathrm{m}^{-3}$ e C-09 0,025 Bq $\cdot \mathrm{m}^{-3}$. Tais valores indicam que o controle de permanência é um fator importante no controle ocupacional dos trabalhadores, assim como o uso de EPI's e os cuidados comportamentais, além do treinamento em radioproteção para permitir o aceso às áreas. Nenhum trabalhador, fiscal ou visitante atingiu o limite de investigação.
\end{abstract}

Palavras-chave: Radiação ionizante (12273), proteção radiológica (12276) e monitoramento de radiação (27784).

\section{INTRODUÇÃO}

A Unidade de Tratamento de Minérios (UTM) pertence às Indústrias Nucleares do Brasil (INB) sendo a sucessora do Complexo Minero-Industrial do Planalto de Poços de Caldas (CIPC), à época operada pela empresa estatal NUCLEBRAS. Recebeu a partir da década de 1980, material radioativo de instalações nucleares de tratamento de areias monazitas para a extração de terras raras [1]. 
O processamento de terras raras começou no Brasil, por iniciativa privada, na década de 50 do século passado. Na década seguinte duas empresas privadas realizavam essas atividades a Mybra e a Orquima, que foram encampadas pela empresa pública Comissão Nacional de Energia Nuclear (CNEN), a qual reuniu as empresas sob o nome de "Administração da Produção da Monazita”. Na década seguinte (1974) as operações saíram da alçada da CNEN e passaram para a NUCLEBRAS, através de sua subsidiária NUCLEBRAS Monazita e Associados (NUCLEMON) [1-2].

Em 1981, os rejeitos gerados no processamento da monazita, antes sob a responsabilidade das empresas particulares, depois da CNEN, começaram a ser encaminhados ao então CIPC, hoje UTM e a sua guarda ficou sob a responsabilidade da então NUCLEBRAS, hoje INB. Esse material é composto basicamente de torta II e mesotório, materiais rico em tório e rádio [2]. Os materiais estão estocados em aproximadamente 20.000 tambores metálicos de $200 \mathrm{l}$ e 20.000 bombonas plásticas de $100 \mathrm{l}$ em seis galpões denominados C-01, C-02, C-05, C-06, C-07 e C-09.

Do ponto de vista radiológico esses galpões são considerados áreas controladas e possuem controle de acesso e são monitorados para verificar a exposição externa e incorporação interna de indivíduos ocupacionalmente expostos (IOE) ou de visitantes e fiscais que necessitam entrar nesses galpões. Para tanto, realiza-se um programa de monitoração radiológica ocupacional que prevê a monitoração de área dos alfa-emissores de meia vida longa presentes no material particulado no ar dos galpões [3]. O presente trabalho visa avaliar a concentração $\left(\mathrm{em} \mathrm{Bq} \cdot \mathrm{m}^{-3}\right.$ ) desses alfa-emissores nos seis galpões da UTM.

\section{MATERIAIS E MÉTODOS}

\subsection{Modelo dosimétrico}

O modelo adotado propõe que a dose efetiva comprometida é proporcional à concentração de atividade dos alfa-emissores de meia vida longa no ar, taxa de respiração e taxa de ocupação e uma constante de proporcionalidade que é o fator de conversão de dose da mistura de radionuclídeos presentes no ar, conforme a equação 1.

$$
D=C \cdot \operatorname{Tr} \cdot \operatorname{To} \cdot \mathrm{e}_{(\mathrm{g}) 1 \mu \mathrm{m}}
$$

onde:

- D é a dose efetiva comprometida, em $\left(\mathrm{Sv} \cdot \mathrm{a}^{-1}\right)$;

- C é a concentração de atividade, em $\left(\mathrm{Bq} \cdot \mathrm{m}^{-3}\right)$;

- Tr é a taxa de respiração, em $\left(\mathrm{m}^{3} \cdot \mathrm{h}^{-1}\right)$; 
- To é a taxa de ocupação, calculada como o produto do número de dias trabalhados por ano, Dt $\left(\mathrm{d} \cdot \mathrm{a}^{-1}\right)$ pelo número de horas trabalhadas por dia, $\mathrm{Ht}\left(\mathrm{h} \cdot \mathrm{d}^{-1}\right)$, em $\left(\mathrm{h} \cdot \mathrm{a}^{-1}\right)$; e

- $\quad \mathrm{e}_{(\mathrm{g}) 1 \mu \mathrm{m}}$ é o fator de conversão de dose da mistura, em $\left(\mathrm{Sv} \cdot \mathrm{Bq}^{-1}\right)$.

Foram considerados 250 dias de trabalho por ano com 8 horas por dia. A taxa de respiração considerada foi estimada em $1,2 \mathrm{~m}^{3} \cdot \mathrm{h}[4]$.

\subsection{Amostragem de ar de alto fluxo}

O equipamento utilizado para amostragem de ar nos galpões é um amostrador de aerossol portátil, composto por um motor com alimentação de corrente alternada de $110 \mathrm{~V}$, um rotâmetro e um suporte para um filtro de fibra de vidro, de alta eficiência de retenção. A vazão de amostragem é de, aproximadamente, $600 \mathrm{l} \cdot \mathrm{min}^{-1}$. Estes amostradores são aferidos por um equipamento de calibração secundária, o qual é calibrado por laboratórios credenciados. As calibrações têm validade estipulada pelo laboratório de calibração sendo preciso verificar se o equipamento está dentro do prazo de validade da calibração [4]. O preparo para uso do amostrador de aerossol consiste em acoplar o filtro de fibra de vidro ao suporte.

O tempo de amostragem é determinado, em cada local de monitoração, através da análise de medidas obtidas anteriormente, visando otimizar o programa de monitoração [4]. O critério utilizado é a obtenção de uma atividade na amostra igual à atividade medida com incerteza relativa de $20 \%$. A relação entre o tempo de amostragem e a concentração no ar medida com incerteza relativa igual a $20 \%$ é dada pela equação (2), abaixo:

$$
C=\frac{F}{2 \cdot V z \cdot T a^{2}} \cdot\left(\frac{1,96}{0,20}\right)^{2} \cdot\left(1+\sqrt{\left(1+\left(\frac{0,20}{1,96}\right)^{2} \cdot 8 \cdot R b \cdot T a\right)}\right)
$$

onde:

- C é a concentração no ar, em $\mathrm{Bq} \cdot \mathrm{m}^{-3}$;

- F é um fator de correção adimensional dado por: $\frac{E f \cdot A b \cdot E r}{60 \cdot F s}$;

o Ef : eficiência de contagem do sistema de radiometria;

o Ab : fator de auto-absorção;

o Er : eficiência de retenção;

o 60 : fator de conversão entre minutos e segundos;

o Fs : área amostrada do filtro

- $\quad \mathrm{Vz}$ : vazão do amostrador de aerossol, $\mathrm{em}^{3} \cdot \mathrm{min}^{-1}$; 
- Ta : tempo de amostragem, em minutos;

- $\mathrm{Rb}$ : contagem do background, em cpm;

O tempo de amostragem é operacionalmente analisado; esse tempo, otimizado para os galpões, é de 15 minutos. O filtro contendo a amostra coletada de aerossol é analisado em um contador alfa total.

\subsection{Concentração alfa total de meia vida longa}

A concentração alfa total de meia vida longa é calculada utilizando-se os resultados das amostragens de aerossol pela fórmula (3), abaixo [5].

$$
C=\frac{1000}{60} \cdot \frac{F s}{E f \cdot A b \cdot E r \cdot V z} \cdot T a \cdot \frac{N}{T} \cdot R b
$$

onde:

- $\mathrm{C}$ : concentração alfa total de meia vida longa, em $\mathrm{Bq} \cdot \mathrm{m}^{-3}$;

- 1000 : fator de conversão entre metro cúbico e litros;

- 60 : fator de conversão entre minutos e segundos;

- Fs : fator de área útil (adimensional), dado por:

$$
\text { o Fs : área amostrada do filtro }
$$

- Ef : eficiência de contagem

- $\mathrm{Ab}$ : fator de auto-absorção

- Er : eficiência de retenção

- $\mathrm{Vz}$ : vazão do amostrador, em $\mathrm{l} \cdot \mathrm{min}^{-1}$

- Ta : tempo de amostragem, em minutos

- $\quad \mathrm{N}$ : número de contagens alfa total

- $\quad \mathrm{T}$ : tempo de contagem alfa total, em minutos

- $\mathrm{Rb}$ : contagem do background, em cpm. 
A contagem alfa de meia vida longa é realizada após 96 horas de intervalo entre o término da amostragem e o início da contagem, para que os alfas emissores de meia vida curta (de frações de segundo a poucas horas) decaiam e não interfiram na contagem.

\section{RESULTADOS E DISCUSSÕES}

O material considerado como fornecedor de material particulado contendo alfa-emissores de meia vida longa foi à torta II. A tabela 1 apresenta os radionuclídeos presentes na torta II, suas proporções, conforme documento da INB [6], suas solubilidades e os fatores de conversão de dose, conforme a posição regulatória PR-003 [7]. Observa-se uma relação entre urânio e tório de $1: 20$.

O fator de conversão de dose da mistura [ $\left.\mathrm{e}_{(\mathrm{g}) 1 \mu \mathrm{m}}\right]$ obtido na tabela 1 permite o cálculo do nível de registro derivado de concentração dos alfa-emissores no ar (C) pela equação (1), usando os dados constantes no modelo dosimétrico (item 2.1).

Tabela 1. Radionuclídeos alfa emissores constantes na torta II, suas proporções na mistura, solubilidades e fatores de conversão de dose [5].

\begin{tabular}{|c|c|c|c|}
\hline Radionuclídeo & Proporção na torta II, em \% & Solubilidade & $\mathbf{e}(\mathrm{g}) 1 \mu \mathrm{m}$ \\
\hline${ }^{232} \mathrm{Th}$ & 20 & M & $4,2 \cdot 10^{-5}$ \\
\hline${ }^{232} \mathrm{Th}$ & 20 & M & $4,2 \cdot 10^{-5}$ \\
\hline${ }^{228} \mathrm{Th}$ & 20 & $\mathrm{M}$ & $3,1 \cdot 10^{-5}$ \\
\hline${ }^{238} \mathrm{U}$ & 1 & $\mathrm{R}$ & $4,9 \cdot 10^{-7}$ \\
\hline${ }^{234} \mathrm{U}$ & 1 & $\mathrm{R}$ & $4,0 \cdot 10^{-5}$ \\
\hline${ }^{230} \mathrm{Th}$ & 1 & M & $3,2 \cdot 10^{-6}$ \\
\hline${ }^{226} \mathrm{Ra}$ & 1 & M & $8,9 \cdot 10^{-7}$ \\
\hline \multicolumn{3}{|c|}{$\mathrm{e}_{(\mathrm{g}) 1 \mu \mathrm{m}}$ da mistura } & $3,34 \cdot 10^{-5}$ \\
\hline $\mathrm{M}=$ moderado & $\mathrm{R}=$ rápido & & \\
\hline
\end{tabular}

Sendo $0,02 \mathrm{~Sv} \cdot \mathrm{a}^{-1} \mathrm{o}$ limite anual de dose efetiva do trabalhador 
Logo, $\mathrm{C}=0,25 \mathrm{~Bq} \cdot \mathrm{m}^{-3}$.

Os valores das concentrações de atividades dos alfa-emissores medidos nas amostras de ar dos seis galpões constam da tabela 2. O limite derivado anual $\left(\mathrm{LD}=0,25 \mathrm{~Bq} \cdot \mathrm{m}^{-3}\right)$ foi superado em 4 galpões (C-06, C-05, C-01 e C-07). A maior média anual de concentração de atividade foi encontrada no galpão C-06 com 8,5 vezes o LD, seguido dos galpões C-05, com 7,9 vezes o LD, C-01 com 4,7 vezes o LD e por fim C-07 com 1,4 vezes o LD. Em um deles (C-09) o valor foi igual ao nível de registro e no galpão C-02 o valor ficou abaixo do nível de registro. A comparação entre as concentrações de atividade média e os limites derivados pode ser visualizada na figura 1.

Tabela 2. Valores das concentrações de atividade de alfa emissores de meia-vida longa nos galpões de materiais radioativos no ano de 2009, em $\mathrm{Bq} \cdot \mathrm{m}^{-3}$.

\begin{tabular}{ccccccc}
\hline \multirow{2}{*}{ Data de coleta } & \multicolumn{7}{c}{ Concentrações de atividades, em Bq· $\mathbf{m}^{-3}$, nos galpões } \\
\cline { 2 - 7 } & C-01 & C-02 & C-05 & C-06 & C-07 & C-09 \\
\hline $22 / 6 / 09$ & 0,77 & 0,01 & 1,7 & 2,12 & 0,20 & 0,02 \\
$21 / 9 / 09$ & 1,56 & 0,002 & 2,25 & 2,15 & 0,48 & 0,03 \\
\hline Média anual & 1,17 & 0,006 & 1,98 & 2,14 & 0,34 & 0,025 \\
\hline Limite derivado & \multicolumn{7}{c}{0,25} \\
\hline
\end{tabular}

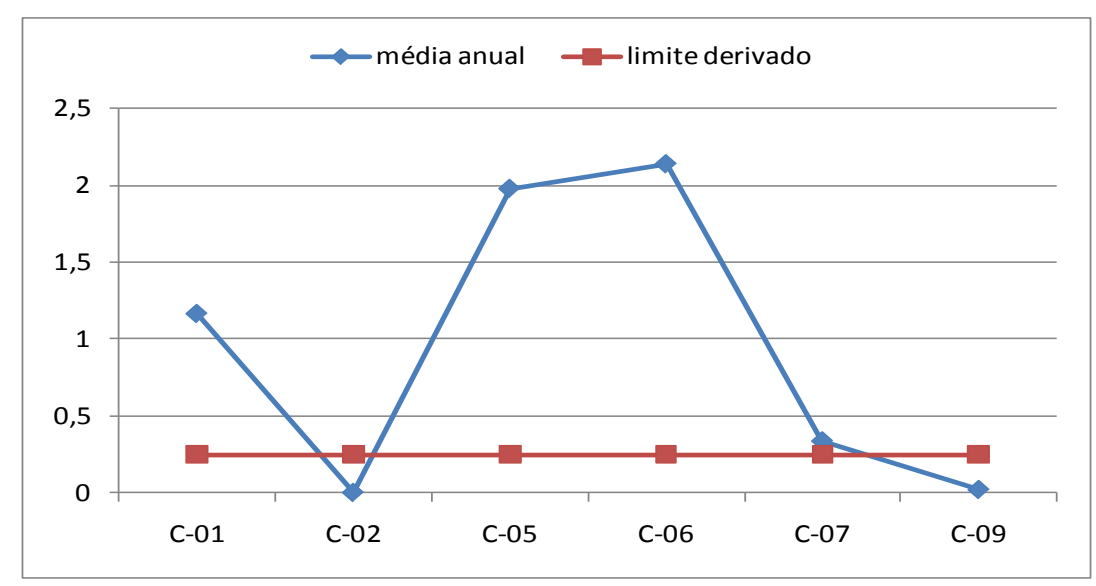

Figura 1. Comparação entre a média anual de concentração de atividade $(\diamond)$ e o limite derivado $(\mathbf{\square})$, nos galpões estudados $\left(\mathrm{Bq} \cdot \mathrm{m}^{-3}\right)$ 
A maioria dos galpões (C-06, C-05, C-01 e C-07) apresenta concentrações de atividade média superior ao LD, fazendo-se necessário o controle de acesso e do tempo de permanência, pelo critério concentração de atividade de alfa-emissores de meia vida longa. No galpão C-06, considerando somente o aspecto inalação de alfa-emissores de meia vida longa, o tempo máximo de permanência para se atingir o limite de dose é estimado em $235 \mathrm{~h} \cdot \mathrm{a}^{-1}$. Para o galpão C-05 esse tempo sobe para $253 \mathrm{~h} \cdot \mathrm{a}^{-1}$; para C-01, são $425 \mathrm{~h} \cdot \mathrm{a}^{-1}$; já o C-07 apresentou um tempo de permanência de $1.429 \mathrm{~h} \cdot \mathrm{a}^{-1}$. O galpão C-09 permite uma jornada de IOE $\left(2.000 \mathrm{~h} \cdot \mathrm{a}^{-1}\right)$. Por fim, em relação ao quesito inalação de alfa-emissores, o galpão C-02, é considerado área livre. Cabe ressaltar, no entanto, que o controle de acesso e o tempo de permanência devem ser avaliados também por outros critérios como, por exemplo, a exposição externa, contaminação de superfície e possibilidade de contaminação.

O treinamento é peça fundamental na manutenção da segurança das operações nos galpões, tanto da equipe do serviço de radioproteção como dos IOE's e outros indivíduos que necessitem ter acesso a esses galpões. Nesse sentido, o controle de permanência é um fator importante no controle ocupacional dos trabalhadores, assim como o uso de EPI's e cuidados comportamentais.

O uso de licença de trabalho assinada pelo supervisor de radioproteção garante a justificação da prática. O estabelecimento de ponto de controle de acesso garante a distribuição de EPI’s e controle do tempo de permanência. O planejamento das atividades é realizado, objetivando a otimização das doses dos trabalhadores. Análise dos registros de acesso aos galpões (dados não relatados aqui) mostra que nenhum IOE, fiscal ou visitante atingiu o limite de investigação nos acessos aos galpões naquele ano.

\section{CONCLUSÕES}

Os galpões C-06, C-05, C-01 e C-07 possuem concentrações de atividade superiores aos limites derivados para alfa emissores de meia vida longa no ar. A magnitude das doses estimadas aponta a necessidade de cuidados de radioproteção para o acesso a esses galpões.

Seguindo o princípio da otimização, o uso de EPI’s, principalmente máscaras com filtros do tipo P-3 (específicos para retenção de radionuclídeos) é imperativo em todos os galpões.

A presença de material particulado contendo alfa-emissores de meia vida longa aponta a necessidade do controle de contaminação removível nos objetos, pisos, maçanetas e outros objetos que podem ter contato com os indivíduos no galpão.

Finalmente, a manutenção dos procedimentos de radioproteção, assim com das monitorações são procedimentos imperativos para o gerenciamento das doses dos indivíduos que necessitem ter acesso aos galpões e são ferramentas que podem ser utilizadas para otimizar as doses efetivas. 


\section{AGRADECIMENTOS}

Os autores gostariam de agradecer a Fundação Nacional de Desenvolvimento do Ensino Superior Particular - FUNADESP, pela bolsa de pesquisa concedida ao Prof. Dr. Wagner de Souza Pereira para a realização desse trabalho.

\section{REFERÊNCIAS}

1. MARTINS, L. A. M. Estado e Exploração Mineral no Brasil. Um Levantamento Básico. Tese de Doutorado em Ciências de Engenharia, Escola Politécnica da Universidade de São Paulo (USP), 1989.

2. CIPRIANI, M. Mitigação dos Impactos Sociais e Ambientais Decorrentes do Fechamento Definitivo de Minas de Urânio. Tese de Doutorado em Ciências na Área de Administração e Política de Recursos Minerais. Instituto de Geociências da Universidade Estadual de Campinas (UNICAMP), 2002.

3. INB - Indústrias Nucleares do Brasil. Programa de proteção radiológica ambiental da Unidade de Tratamento de Minério, condição de área parada, 2002, 23 pp.

4. INB - Indústrias Nucleares do Brasil. Monitoração de área por meio de amostragem de aerossol. Instrução Operacional da UTM. IO-UTM-PO-54, 2010, 16 pp.

5. INB - Indústrias Nucleares do Brasil. Programa de proteção radiológica ambiental da Usina de Interlagos, 2010, $45 \mathrm{pp}$.

6. CNEN - Comissão Nacional de Energia Nuclear. Posição regulatória 3.01 / 003 - Coeficientes de dose para indivíduos ocupacionalmente expostos, 2005, 52 pp.

7. INB - Indústrias Nucleares do Brasil. Análise radiométrica de amostras. Instrução operacional da USIN. IO-COSAP-PO-12. 2010, 12 pp. 